\title{
Solvent Effect on the Allylic Oxidation of Cyclohexene Catalyzed by Nitrogen Doped Carbon Nanotubes
}

\author{
Yonghai Cao, Hao Yu*, Hongjuan Wang, Feng Peng* \\ School of Chemistry and Chemical Engineering, South China University of \\ Technology, Guangzhou, Guangdong, 510640 (China)
}

* Tel. \& Fax: +86 2087114916.
E-mail address: yuhao@ scut.edu.cn (H. Yu); cefpeng@ @scut.edu.cn (F. Peng). 


\section{Abstract}

A wide spectrum of, up to 22 , organic solvents, including aprotic/protic aliphatics/aromatics, were studied to achieve a comprehensive understanding to the solvent effect on the cyclohexene oxidation. It was found that the catalytic activity was significantly influenced by the viscosity, polarity and basicity of solvents in this reaction. Among these solvents, the polar aprotic aliphatic solvents displayed higher catalytic reactivities than other types of solvents. A volcano curve was found describing the dependence of activity on solvent basicity because of the suitable stability of intermediates. Among the solvents investigated, acetonitrile afforded the highest activity and selectivity of 2-cyclohexen-1-one, because of a good compromise between strong polarity and moderate basicity.

Keywords: Selective oxidation; cyclohexene; solvent effect; carbon nanotubes; 2-cyclohexen-1-one; nitrogen doping 


\section{Introduction}

The selective oxidation of hydrocarbons towards oxygenated derivatives is a significant chemical value-chain, through which the relatively cheap molecules can be converted into value-added products [1-3]. Numerous studies have demonstrated that solvents display a dramatic influence on the catalytic activity and products distributions, especially for the oxidation of alkenes [2, 4-7]. The solvent properties, such as polarity, dielectric constant and basicity, influenced the activity of the epoxidation of styrene, 1 -hexene and cyclohexene over titanosilicate catalysts $[6,8]$. In addition, a synergistic effect between the solvent property and the hydrophilicity-hydrophobicity of the zeolite also affected the catalytic activity, as observed in the case of 1-hexene oxidation towards the production of alcohol and 2-cyclohexen-1-one [9]. Fan et al. investigated the solvent effects on the activity, epoxide selectivity, and $\mathrm{H}_{2} \mathrm{O}_{2}$ efficiency in the liquid-phase epoxidation of 1-hexene and cyclohexene over three types of zeolites (TS-1, Ti-Beta, and Ti-MWW) [6].

The literature results indicate that solvents may interplay with the active sites on catalyst surfaces, thereby modify the reaction pathway and the distribution of products in the alkene oxidation reactions $[6,10]$. However, the manner of solvent affecting the reaction might be changed as the catalyst is upgraded. Nanocarbons have attracted great attentions from the research and industrial communities in the past two decades as metal-free catalysts, due to their unique chemical and physical properties [11-13]. Recently, nanocarbons, especially nitrogen doped carbon nanotubes (NCNTs), displayed excellent performance in the allylic oxidation of cyclohexene, affording a 
high selectivity of 2-cyclohexene-1-none [14]. The remarkable activity can be explained by the stabilization of peroxyl and cycloxyl radicals and the charge transfer between NCNTs and radicals, which is facilitated by delocalized electrons in graphene layers [14-16]. The significant solvent effect in the selective oxidation of hydrocarbons catalyzed by carbons has been observed. For instance, Yang et al. found that the unique catalytic efficiency of the hydroxylation of benzene catalyzed by graphene in immiscible water as solvent, because the products can be rapidly transferred to solvent to avoid the over-oxidation [5]. Our very recently work also revealed that solvents could greatly influence the activity of $\alpha$-pinene oxidation over CNTs [17]. Despite these achievements, a comprehensive understanding to the solvent effect on the alkene oxidation is so far unavailable, because the library of solvents was usually too small to uncover the general tendency of solvent effect. Moreover, the synergy between solvents and carbon catalysts has not been revealed.

In this work, we have studied the effect of solvents on the allylic oxidation of cyclohexene catalyzed by NCNTs. To achieve an in-depth understanding, 22 organic solvents were investigated, which are categorized into four types, namely protic/aprotic aliphatics/aromatics, according to their properties and structures. By correlating the catalytic performance with the solvent properties, i.e. viscosity, polarity and scale of basicity, the solvent effect could be rationalized.

\section{Experimental}

\subsection{Preparation of catalysts}

The NCNTs were produced according to our previous works [14, 18]. In brief, 
the NCNTs were synthesized by a CVD method using aniline as carbon and nitrogen sources. The growth of NCNTs was carried out at $800{ }^{\circ} \mathrm{C}$ in $\mathrm{NH}_{3}$ at $500 \mathrm{Ncm}^{3} \mathrm{~min}^{-1}$. The metal impurities were removed by concentrated $\mathrm{HCl}$ for $4 \mathrm{~h}$ before catalytic tests.

\subsection{Cyclohexene oxidation}

The cyclohexene oxidation reaction was carried out as same as the previous work [14]. 22 solvents were used in this work and their physical/chemical properties are summarized in Table S1. Typically, $10 \mathrm{~mL}$ cyclohexene, $20 \mathrm{~mL}$ solvent, $2 \mathrm{~mL}$ o-dichlorobenzene (o-DCB) as internal standard and $50 \mathrm{mg}$ catalyst were charged into the autoclave. Before reaction, the reactor was flushed with $\mathrm{N}_{2}$. Then, the reactor was heated to $80{ }^{\circ} \mathrm{C}$, and subsequently pure $\mathrm{O}_{2}$ was fed into the reactor $(1 \mathrm{MPa}, 4 \mathrm{~h})$. The products were analyzed by gas chromatography.

\section{Results and discussion}

\subsection{Effect of viscosity}

The viscosity of solvent plays significant roles in the liquid-phase reactions through altering the mass transfer, resulting in the different activity [19]. In this work, the solvent viscosity varies across a wide range. The effect of viscosity on the cyclohexene conversion is partially presented in Fig.1. Within the scope of solvent investigated, there is not universal tendency showing a common effect of viscosity on activity. Fig. 1 shows a serious decrease of conversion with increasing viscosity in the case of protic $-\mathrm{OH}$ containing solvents, probably due to the negative effect of viscosity on mass transfer in liquid phase. However, the dependence of conversion on the viscosity of $-\mathrm{C}=\mathrm{O}$ group containing solvent is quite weak. Similar situation can be 
observed for other solvents, such as the aprotic aliphatic/aromatics solvents, displaying the opposite tendency in the cyclohexene oxidation between those two kinds of solvents (not shown). These inconsistent even opposite responses suggest that the viscosity has minor influence on the cyclohexene oxidation, but is not the essential factor under current conditions. Therefore, we focus on the effects of specific physical/chemical property of solvents, for instance polarity and basicity, on the cyclohexene oxidation.

\subsection{Effect of polarity}

Polarity of solvent has a significant influence on the reactivity in the cyclohexene oxidation, since it plays a significant role on stabilizing the transition state (intermediate radical species) and the proton transfer [20-22]. In this work, the effect of polarity of protic/aprotic aliphatics/aromatics solvents were investigated, respectively. The dependences of cyclohexene conversion on polarity are plotted in Fig. 2. For the four types of solvents, the conversion increased along with the polarity, indicating that solvents with higher polarity are favorable for the catalytic efficiency. Clear pseudo-linear relationships between polarity and conversion were observed for aprotic and protic aliphatics. It may be attributed to the enhanced solubility of polar oxides produced in polar solvents. The solvents with strong polarity probably enhanced the desorption of polar oxide products to avoid blocking the active sites on NCNTs $[4-5,17]$. Some exceptional results are observed, e.g. acetone, chloroform and acetonitrile, with large deviations from other aprotic aliphatics. Acetone is probably unstable under the current reaction conditions, because it may be decomposed to 
acetic acid as a protic solution suppressing the reactivity. The higher activities in chloroform and acetonitrile may be attributed to their lower viscosities (see Table S1). Nevertheless, the dependence of conversion on polarity demonstrates that polarity may be a good indicator to a rational selection of reaction solvents, which may lead to a huge difference of reactivity from $20 \%$ to $60 \%$ conversion under the same conditions.

Within the aromatic solvents investigated, there is not clear tendency describing the dependence of cyclohexene conversion on polarity. In general, aromatics exhibits lower conversions than the aliphatic solvents. This is probably because the conjugated competitive adsorption of solvent and cyclohexene upon NCNTs, since aromatics molecules may strongly interact with sp2 carbon systems [14, 23]. For example, toluene can strongly adsorb on and block active sites [17]. It could be argued that aromatic solvents might be reactive on the benzyl position. However, our previous work demonstrated that the oxidation of ethyl benzene or aromatic solvents needs more severe conditions, such as high temperatures $\left(>145^{\circ} \mathrm{C}\right)$ and pressures $(>1.5$ $\mathrm{MPa}$ ), without additives or co-reactants [24]. Hence, in this study, the lower reactivity in aromatics could be mainly attributed to the competitive adsorption between substrate and toluene upon active sites of NCNTs.

It can be clearly observed that the aprotic solvents displayed higher reactivity than the protic ones in this study. This behavior could be rationalized through analyzing the mechanism of cyclohexene oxidation catalyzed by NCNTs, as summarized by the following equations (1-10) [2-3, 14, 25-27]: 
Initiation: $\quad \mathrm{RH}+$ initiator $\rightarrow \mathrm{R} \bullet+\mathrm{H}$-initiator

Propagation: $\mathrm{R} \bullet+\mathrm{O}_{2} \rightarrow \mathrm{ROO} \bullet$

$$
\begin{aligned}
& \mathrm{ROO} \bullet+\mathrm{RH} \rightarrow \mathrm{ROOH}+\mathrm{R} \bullet \\
& \mathrm{ROOH} \rightarrow \mathrm{RO} \bullet+\bullet \mathrm{OH} \\
& \mathrm{RO} \bullet+\mathrm{RH} \rightarrow \mathrm{ROH}+\mathrm{R} \bullet \\
& \bullet \mathrm{OH}+\mathrm{RH} \rightarrow \mathrm{H}_{2} \mathrm{O}+\mathrm{R} \bullet \\
& \mathrm{RO} \bullet+\mathrm{O}_{2} \rightarrow \mathrm{R}=\mathrm{O}+\mathrm{HO}_{2} \bullet
\end{aligned}
$$

In this process, the abstraction of the weakly bonded $\alpha-\mathrm{H}$ atom and the stabilization of radical species play key roles. In our previous study, the radical nature of the carbon-catalyzed oxidation of cyclohexene has been verified [14]. The participation of reactive radicals is a prerequisite of the catalytic role of NCNTs. Nanocarbons can greatly enhance the radical propagation (equations 3-7) by elongating the lifetime of radicals through forming carbon-radical complexes, thus accelerate the overall reaction [14-15, 17-18]. The donor-acceptor interaction between the solvent and intermediate radical species affects the abstraction and transfer of hydrogen atoms, thereby influences the reactivity [28-29]. The protic solvents afford protons in the system through the proton-donation behavior, which may suppress the $\alpha-\mathrm{H}$ atom abstraction, therefore resulting in a low reactivity [9]. 


\subsection{Effect of basicity}

Above analysis suggests a dependence of reactivity on the hydrogen-bond donation/acceptance ability of solvents. The parameters $\gamma$ (hydrogen-bond donor) and $\delta$ (hydrogen-bond acceptor) refer to donation and acceptance of protons involved in hydrogen bonding [7]. In this paper, we use basicity, instead of $\delta$, as an alternative indicator reflecting the solvent's hydrogen-bond acceptor basicity scale $(\beta)$ to analyze the dependence, because of the higher availability of basicity data [7]. For the selective oxidation, the basicity of solvent can significantly influence the reactivity [30-31]. For example, Russel found that the solvent with higher basicity afford higher stability of $\pi$-complex, therefore giving better performance in the chlorination reactions [30]. Fig. 3 shows the effects of basicity on the cyclohexene conversion. Two clear and similar volcano-like curves can be used to describe the dependence of reactivity on $\beta$ parameter for aliphatics and aromatics, respectively. In the cases of aliphatics, the cyclohexene conversion displayed maximum at basicity of $\sim 0.3$ for acetonitrile. Although aromatic solvents showed inferior reactivity compared with the aliphatics, it is interesting that the maximum conversion appears at the similar $\beta$ value. These results indicate that the modest basicity of solvent is beneficial for the cyclohexene oxidation. It is probably because a suitable stability of reactive-intermediate-NCNT complex is needed for the oxidation. The highest activity was obtained using acetonitrile as solvent in this study. Two explanations can be presented: (1) acetonitrile has strong polarity and can well desorb the polar products; (2) the basicity of acetonitrile is very suitable for the radical chain propagation. 


\subsection{Solvent effect on product selectivity}

In the selective oxidation of cyclohexene catalyzed by NCNTs, 5 kinds of oxygenated derivates are produced, among which 2-cyclohexen-1-one (-one) and 2-cyclohexen-1-ol (-ol) are dominant [14]. Herein, we correlate the selectivity of -one and -ol with the solvent parameters by fixing the cyclohexene conversion at around $25 \%$ to avoid the effect of reaction extent, which provided us a clear observation for the distribution of products selectivities (Table 1).

Table 1. Selectivity patterns of cyclohexene oxidation catalyzed by NCNTs in various solvents.

\begin{tabular}{|c|c|c|c|c|c|c|c|}
\hline & \multirow[b]{2}{*}{ Solvents } & \multicolumn{6}{|c|}{ Selectivity (\%) } \\
\hline & & & & & & & \\
\hline \multirow{11}{*}{$\begin{array}{l}\text { aprotic } \\
\text { aliphatics }\end{array}$} & Tetrachloromethane & 15.9 & 11.3 & 29.0 & 1.8 & 33.5 & 91.4 \\
\hline & n-Hexane & 3.9 & 16.6 & 32.8 & 2.2 & 34.1 & 89.5 \\
\hline & Cyclohexane & 3.3 & 14.1 & 31.6 & 1.2 & 42.9 & 93.0 \\
\hline & Trichloromethane & 6.6 & 12.7 & 29.4 & 2.5 & 40.8 & 92.0 \\
\hline & Acetonitrile & 4.7 & 11.8 & 39.5 & 3.2 & 38.4 & 97.5 \\
\hline & 1,2-Dichloroethane & 3.8 & 7.3 & 17.4 & 2.2 & 62.1 & 92.7 \\
\hline & 1,4-Dioxane & 4.3 & 6.2 & 32.8 & 1.1 & 44.6 & 89.1 \\
\hline & Acetone & 4.6 & 7.2 & 31.4 & 0.7 & 50.7 & 94.6 \\
\hline & 2-Butanone & 3.6 & 7.1 & 26.8 & 2.7 & 49.8 & 90.0 \\
\hline & 4-Methyl-2-pentanone & 3.1 & 6.9 & 21.4 & 0.2 & 51.9 & 83.4 \\
\hline & Cyclohexanone & 5.0 & 7.9 & 23.0 & 2.5 & 48.3 & 86.7 \\
\hline \multirow{3}{*}{$\begin{array}{c}\text { protic } \\
\text { aliphatics }\end{array}$} & n-Heptanol & 0.4 & 0.9 & 34.7 & 14.1 & 35.1 & 85.1 \\
\hline & 1-Octanol & 3.9 & 7.8 & 32.7 & 3.2 & 48.1 & 95.7 \\
\hline & 2-Propanol & 4.5 & 5.4 & 34.4 & 1.7 & 47.9 & 93.9 \\
\hline
\end{tabular}




\begin{tabular}{cccccccc} 
& Methanol & 2.0 & 7.7 & 36.2 & 2.2 & 34.9 & 82.9 \\
& Ethanol & 6.1 & 9.2 & 34.9 & 1.0 & 46.7 & 97.8 \\
\hline \multirow{2}{*}{ aprotic } & Toluene & 8.9 & 12.1 & 25.3 & 2.4 & 34.3 & 83.0 \\
aromatics & p-Xylene & 6.3 & 11.0 & 33.5 & 4.7 & 36.5 & 92.0 \\
& 1,3,5-Trimethylbenzene & 4.3 & 8.4 & 25.4 & 2.5 & 43.4 & 84.0 \\
& Ethylbenzene & 5.1 & 10.1 & 39.6 & 3.1 & 40.4 & 98.4 \\
& Acetophenone & 3.1 & 15.3 & 18.7 & 1.4 & 57.8 & 96.3 \\
\hline protic & & & & & & & \\
aromatics & Benzyl alcohol & 4.0 & 10.6 & 38.9 & 4.9 & 37.3 & 95.7 \\
\hline
\end{tabular}

Compared to activity, the solvent effect on selectivity is much more complex, because the selectivity pattern is determined by the complicated interplay between solvent and multiple compounds and intermediates. In the cyclohexene oxidation, cyclohexene is oxidized by molecular oxygen via allylic oxidation and epoxidation routes $[3,14]$. NCNTs can efficiently boost the yield of -one by stabilizing peroxy radicals and improving the conversion of alkoxyl radicals to ketone [3, 14]. Especially, the higher ketone/alcohol ratios than that in the blank experiment (1.2 5.2 vs. 0.6) were observed for most of the solvents (Table 1), indicating the involving of NCNTs for the production of ketone (equations 7) [14]. In this work, we used the selectivity of -one and -ol to indicate the effect of solvent on selectivity. As shown in Fig. 4, in general, there are relatively weak dependences of the selectivity of -one and -ol on polarity, suggesting the similar reaction pathway in different solvents. For protic aliphatics and most of aprotic aliphatics, the selectivity of -one slightly increased with increasing polarity, while the selectivity of - ol slightly declined, resulting in an increase of -one/-ol ratio with polarity. Scattering -one selectivities are observed in 
aprotic aromatics, probably due to the complicated competitive adsorption among multi components as mentioned above. Solvents with $-\mathrm{C}=\mathrm{O}$ functionality, i.e. acetophenone, cyclohexanone, 4-methyl-2-pentanone and butanone, displayed relatively lower -one selectivities. Meanwhile, the selectivity of cyclohexyl hydroperoxide was relatively high in these cases, indicating that the decomposition of hydroperoxide was inhibited in these keto-solvents. These results suggested that the ketone compounds as solvent may suppress equations 4, 7 and 9, where peroxide species are involved to yield ketone. Similarly, dichloromethane showed a low selectivity of - one and high cyclohexyl hydroperoxide selectivity. We speculate that dichloromethane solvent may cover the active sites on the surface of NCNTs, thereby suppress the cleavage of cyclohexyl hydroperoxide to produce -one [32]. On current stage, it is still lack of the knowledge on the effect of solvent molecular configuration on selectivity.

\section{Conclusions}

In conclusion, the solvent effects on the selective oxidation of cyclohexene with NCNTs as catalyst have been revealed by analyzing the reactivity and selectivity across a solvent library containing up to 22 organic solvents. The higher polarity is beneficial for the activity in protic and aprotic aliphatics, because of the improved desorption of oxide products. A modest solvent basicity is required for high reactivity, implying that a suitable stability of intermediates is needed. On the aspect of selectivity, the increase of solvent polarity results in a slight increase of -one selectivity and decrease of -ol selectivity, thus a higher ketone/alcohol ratio. In 
addition, the selectivity of oxygenated products strongly depends on the functionality of solvent. The comprehensive phenomenological survey may guide a more rational design of the liquid-phase oxidation reactions catalyzed by nanocarbons, the emerging metal-free catalysts.

\section{Acknowledgements}

This work was supported by the National Science Foundation of China (Nos. 21503082, 21133010 and 21273079), Guangdong Provincial Natural Science Foundation (Nos. S20120011275 and 2014A030310447), Program for New Century Excellent Talents in University (NCET-12-0190), Fundamental Research Funds for the Central Universities of China (Nos. 2015PT012 and 2015ZM048). 


\section{References}

[1] Sul'pin, G. B., Organic Reactions Catalyzed by Metal Complexes. Nauka: Moscow, 1988.

[2] Sheldon, R. A.; Kochi, J. K., Metal-catalyzed oxidations of organic compounds. Academic Press: New York, 1981.

[3] Neuenschwander, U.; Guignard, F.; Hermans, I., ChemSusChem 3 (2010) 75.

[4] Luo, J.; Peng, F.; Yu, H.; Wang, H. J., Chem. Eng. J. 204 (2012) 98.

[5] Yang, J.-H.; Sun, G.; Gao, Y. J.; Zhao, H. B.; Tang, P.; Tan, J.; Lu, A.-H.; Ma, D., Energ. Environ. Sci. 6 (2013) 793.

[6] Fan, W.; Wu, P.; Tatsumi, T., J. Catal. 256 (2008) 62.

[7] Reichardt, C.; Welton, T., Solvents and solvent effects in organic chemistry. John Wiley \& Sons: Weinheim, 2011.

[8] Kumar, S. B.; Mirajkar, S. P.; Pais, G. C. G.; Kumar, P.; Kumar, R., J. Catal. 156 (1995) 163.

[9] Corma, A.; Esteve, P.; Martınez, A., J. Catal. 161 (1996) 11.

[10] Lignier, P.; Mangematin, S.; Morfin, F.; Rousset, J.-L.; Caps, V., Catal. Today 138 (2008) 50.

[11] Su, D. S.; Zhang, J.; Frank, B.; Thomas, A.; Wang, X.; Paraknowitsch, J.; Schlögl, R., ChemSusChem 3 (2010) 169.

[12] Wang, Y.; Wang, X.; Antonietti, M., Angew. Chem. Int. Ed. 51 (2012) 68.

[13] Su, D. S.; Perathoner, S.; Centi, G., Chem. Rev. 113 (2013) 5782.

[14] Cao, Y.; Yu, H.; Peng, F.; Wang, H., ACS Catal. 4 (2014) 1617.

[15] Cao, Y.; Yu, H.; Tan, J.; Peng, F.; Wang, H.; Li, J.; Zheng, W.; Wong, N.-B., Carbon 57 (2013) 433.

[16] Yang, X. X.; Wang, H. J.; Li, J.; Zheng, W. X.; Xiang, R.; Tang, Z. K.; Yu, H.; Peng, F., Chem. Eur. J. 19 (2013) 9818.

[17] Cao, Y.; Li, Y.; Yu, H.; Peng, F.; Wang, H., Catal. Sci. Technol. 5 (2015) 3935.

[18] Yu, H.; Peng, F.; Tan, J.; Hu, X. W.; Wang, H. J.; Yang, J. A.; Zheng, W. X., Angew. Chem. Int. Edit. 50 (2011) 3978.

[19] Mukherjee, S.; Vannice, M. A., J. Catal. 243 (2006) 108.

[20] Mitroka, S.; Zimmeck, S.; Troya, D.; Tanko, J. M., J. Am. Chem. Soc. 132 (2010) 2907.

[21] Hasegawa, E.; Xu, W.; Mariano, P. S.; Yoon, U. C.; Kim, J. U., J. Am. Chem. Soc. 110 (1988) 8099.

[22] Yoon, U. C.; Kim, J. U.; Hasegawa, E.; Mariano, P. S., J. Am. Chem. Soc. 109 (1987) 4421.

[23] Li, X.-H.; Wang, X.; Antonietti, M., ACS Catal. 2 (2012) 2082.

[24] Luo, J.; Peng, F.; Yu, H.; Wang, H. J.; Zheng, W. X., ChemCatChem 5 (2013) 1578 .

[25] Cavani, F.; Teles, J. H., ChemSusChem 2 (2009) 508.

[26] Franz, G.; Sheldon, R. A., Oxidation-Ullmann's Encyclopedia of Industrial Chemistry. Wiley-VCH Verlag GmbH \& Co. KGaA: Weinheim, 2000.

[27] Emanuel, N. M.; Denisov, E. T.; Maizus, Z. K., Liquid phase oxidation of 
hydrocarbons. Plenum: New York, 1967.

[28] Roberts, B., Chem. Soc. Rev. 28 (1999) 25.

[29] Hazarika, S.; Goswami, P.; Dutta, N. N., Chem. Eng. J. 94 (2003) 1.

[30] Russell, G. A., J. Am. Chem. Soc. 80 (1958) 4987.

[31] Litwinienko, G.; Ingold, K., Acc. Chem. Res 40 (2007) 222.

[32] Maldotti, A.; Bartocci, C.; Varani, G.; Molinari, A.; Battioni, P.; Mansuy, D., Inorg. Chem. 35 (1996) 1126. 
Figures and captions

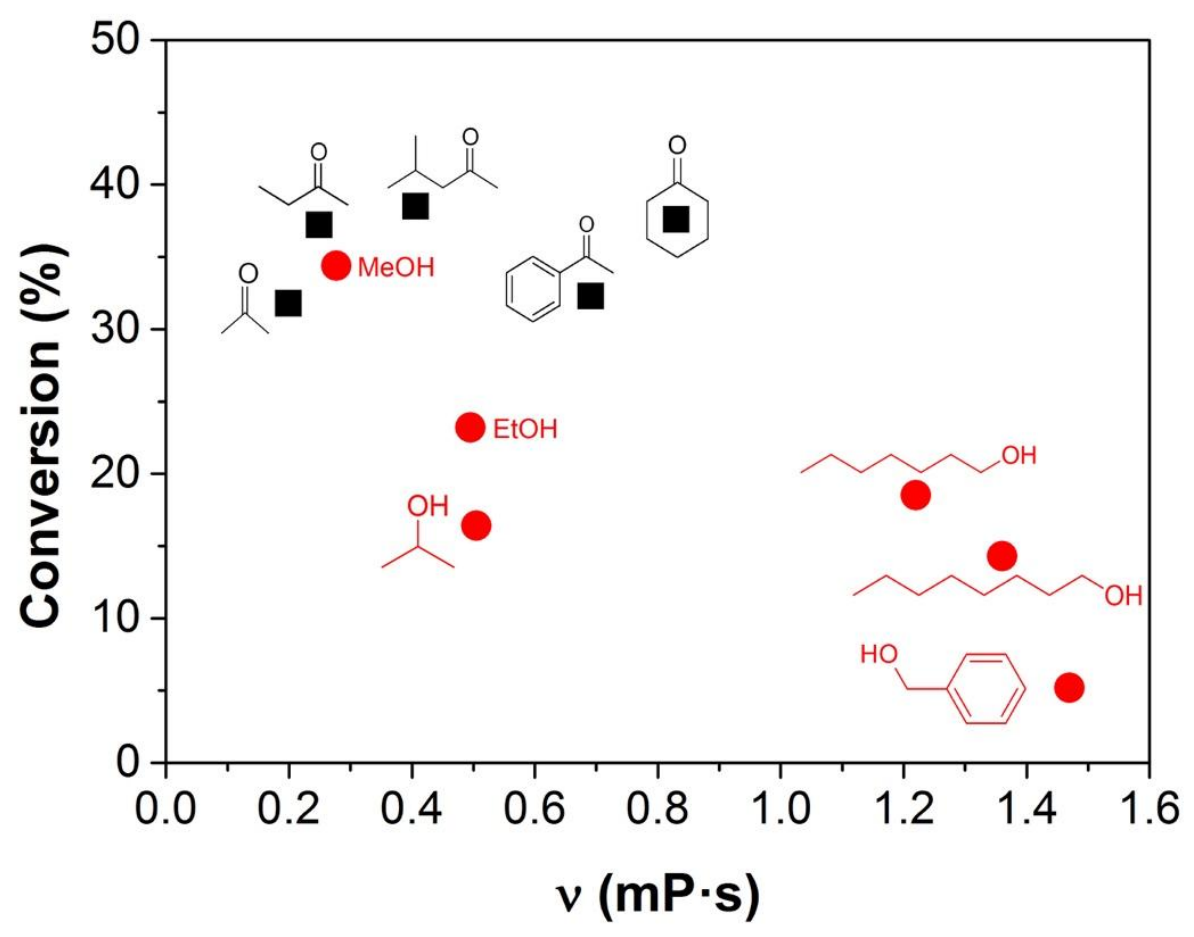

Fig. 1. Effect of solvent viscosity on the conversion of cyclohexene. 


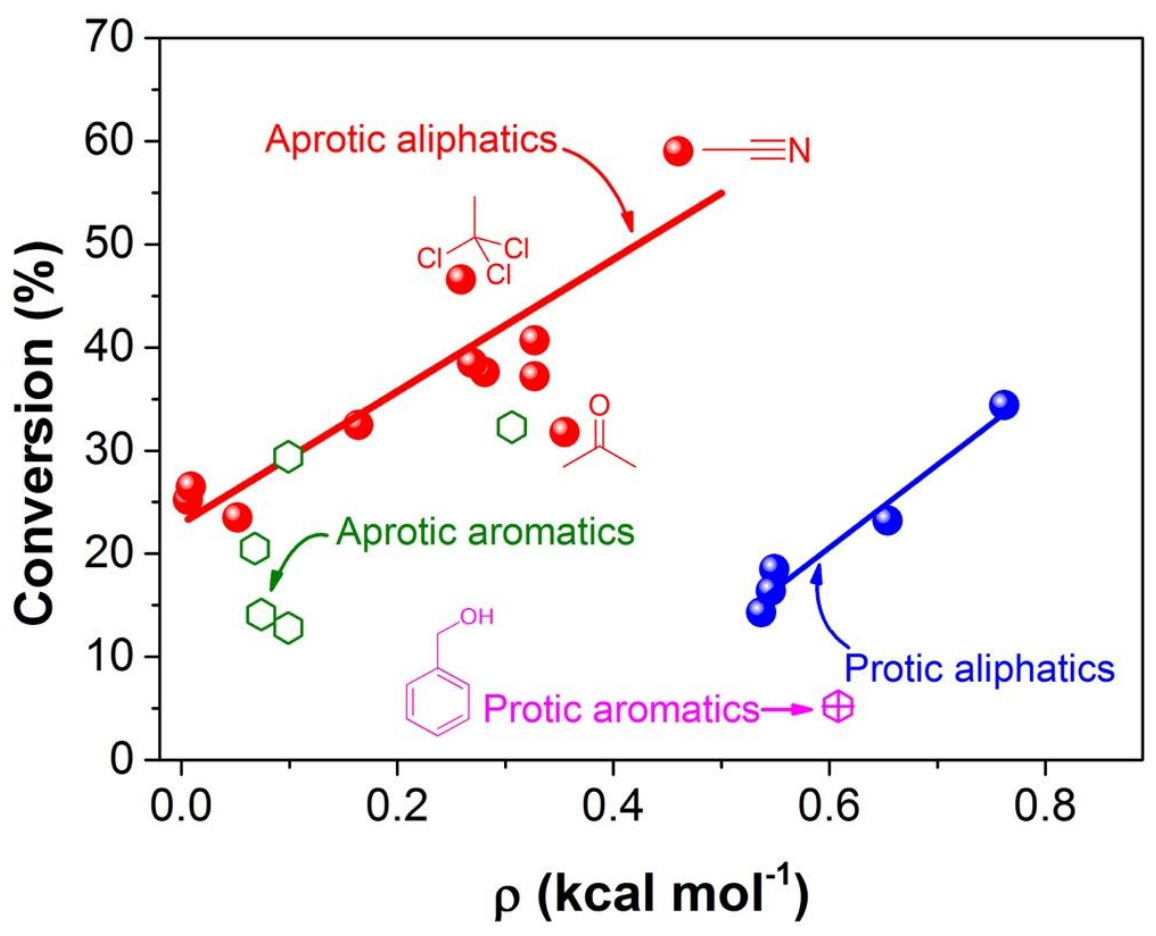

Fig. 2 Effect of solvent polarity on the conversion of cyclohexene. 


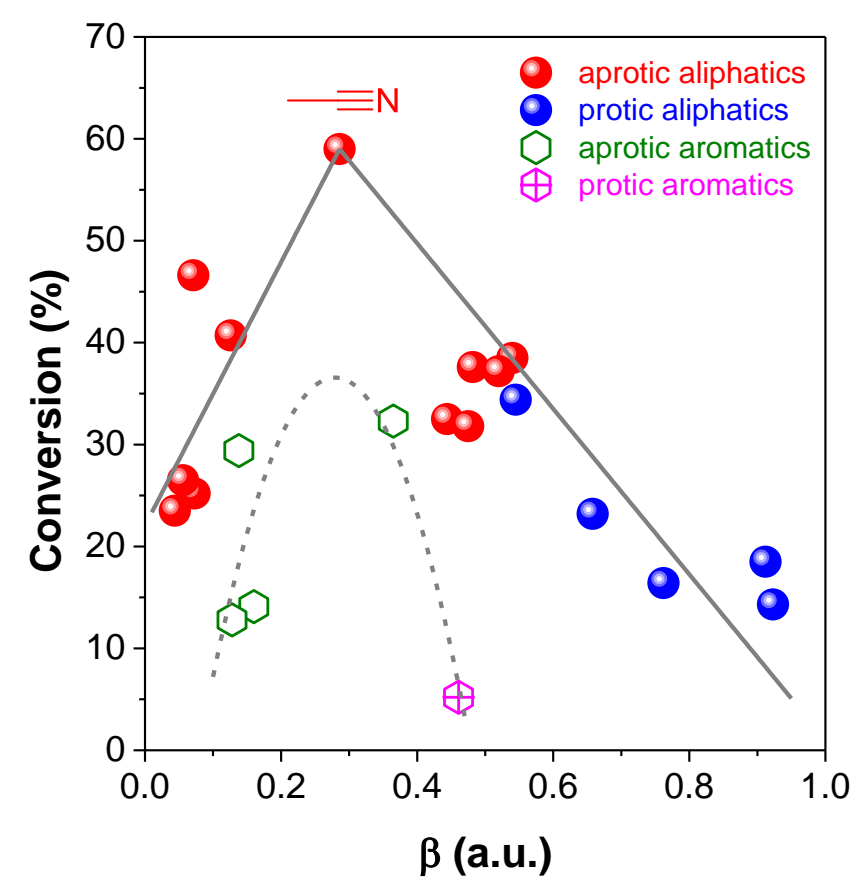

Fig. 3. Effect of solvent basicity on the conversion of cyclohexene. 


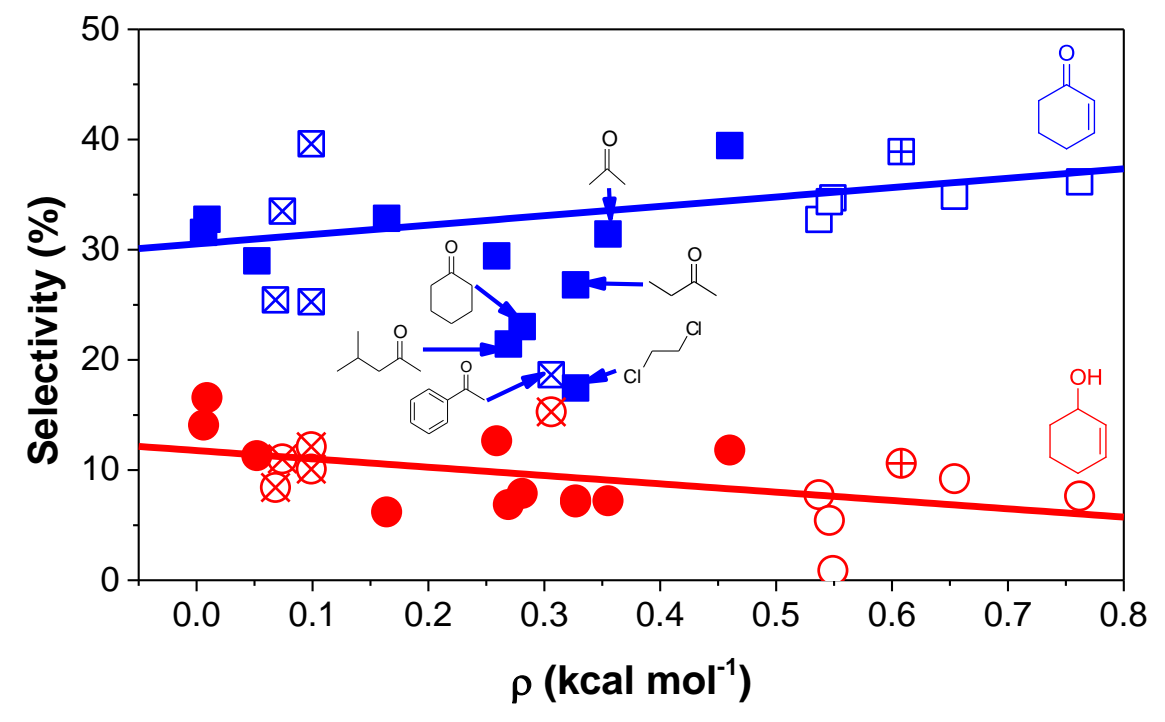

Fig. 4 Effect of solvent polarity on the selectivity of 2-cyclohexen-1-one and 2-cyclohexen-1-ol at about 25\% conversion. Legend: -one selectivity in ( $\boldsymbol{\square})$ aprotic aliphatics, $(\square)$ protic aliphatics, $(\otimes)$ aprotic aromatics and $(\boxplus)$ protic aromatics; -ol selectivity in $(\bullet)$ aprotic aliphatics, $(\bigcirc)$ protic aliphatics, $(\otimes)$ aprotic aromatics and $(\oplus)$ protic aromatics. 


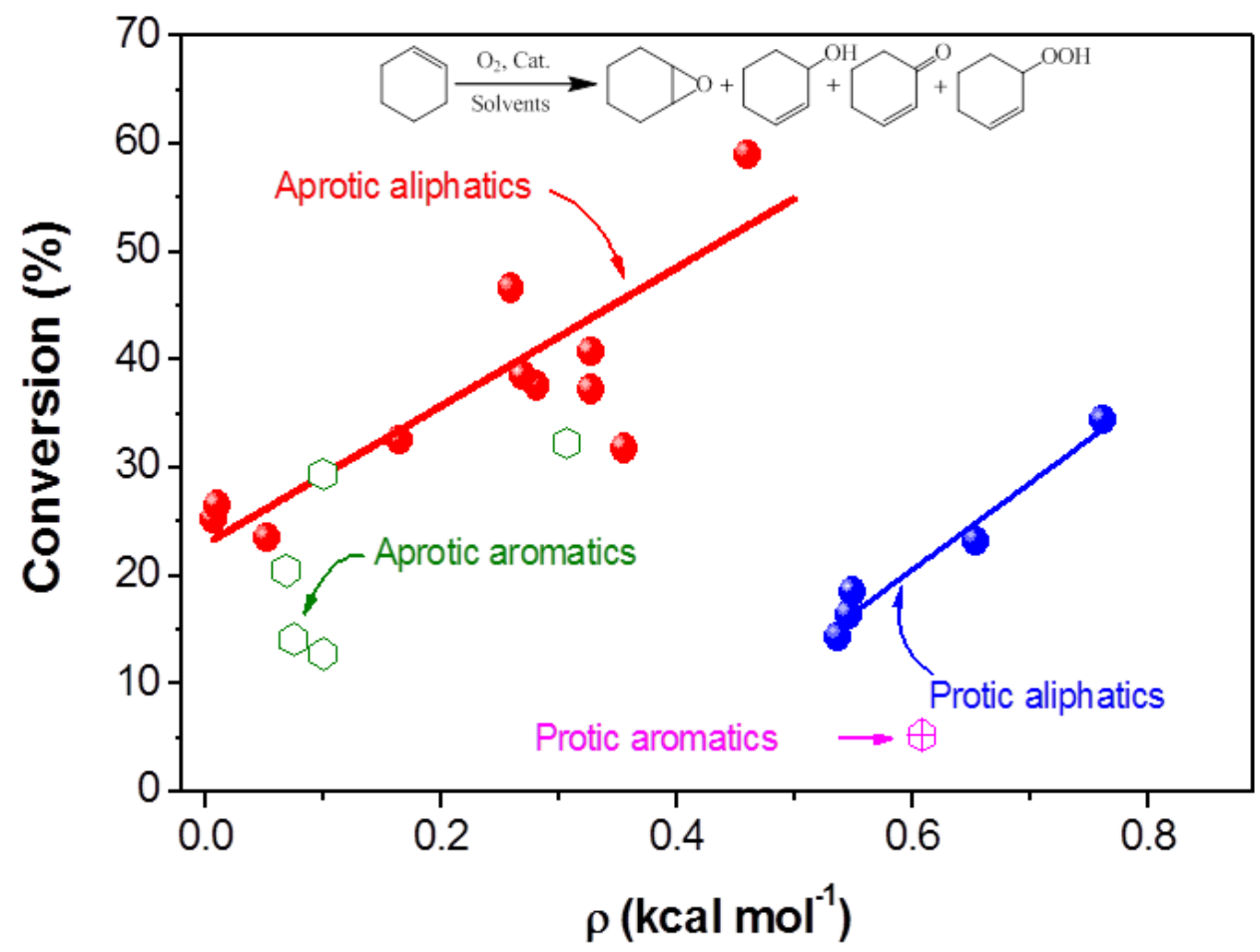

